\title{
El retorno a la universidad durante la pandemia: perspectivas de estudiantes de obstetricia de una universidad pública
}

Jennifer Rojas-Vega 1,a ; Diayan Castro-Gómez 1,a; Valery Damacén-Oblitas 1,a; Jessica Rojas-Silva 2,a; Victor MoquillazaAlcántara*3,b

\section{RESUMEN}

Objetivo: Determinar la perspectiva del estudiante de obstetricia respecto al retorno de las actividades universitarias teóricas y prácticas en un contexto de pandemia.

Materiales y métodos: Estudio analítico transversal, en el que participaron 115 estudiantes matriculados en la carrera de obstetricia (del segundo al quinto año) de una universidad pública de Lima. Se evaluó la proporción de estudiantes que están de acuerdo con el retorno a las actividades teóricas y prácticas, los recursos con los que cuentan y su situación familiar. Las asociaciones ajustadas fueron evaluadas mediante regresión de Poisson.

Resultados: El 58,26 \% [IC95 \%: 48,93-67,03 \%] de los participantes está de acuerdo con el retorno a las actividades teóricas virtuales; y el 6,09 \% [IC95\%: 2,89-12,34\%], con el regreso a las prácticas hospitalarias. Solo el 66,09\% cuenta con internet, dispositivo móvil y un ambiente adecuado de estudio en su hogar. El grupo que no estuvo de acuerdo con el retorno a la práctica hospitalaria se caracterizó por presentar un antecedente patológico personal $(p<0,001)$ y convivir con dos o más personas mayores de 65 años $(p<0,001)$. Los principales antecedentes clínicos de los familiares mayores de 65 años fueron hipertensión arterial $(36,89 \%)$, diabetes $(19,04 \%)$ y obesidad $(15,47 \%)$.

Conclusiones: Es escasa la proporción de estudiantes de obstetricia que están a favor del retorno a los hospitales, motivados principalmente por tener antecedentes patológicos personales y convivir con familiares mayores de 65 años. Cerca de la mitad de los participantes cuenta con recursos digitales que permiten su aprendizaje remoto.

Palabras clave: COVID-19; Educación profesional; Pandemias; Práctica profesional (Fuente: DeCS BIREME).

\section{Returning to the university during the pandemic: perspectives of midwifery students from a public university}

ABSTRACT

Objective: To determine the perspectives of midwifery students regarding the return to the theoretical and practical university activities in the context of a pandemic.

Materials and methods: A cross-sectional analytical study involving 115 second- to fifth-year midwifery students from a public university in Lima. The number of students who agreed to return to the theoretical and practical activities, the resources they had and their family situation were evaluated. Adjusted associations were estimated using Poisson regression.

Results: Fifty-eight point two six percent (58.26\%) [95\% Cl: $48.93-67.03 \%$ ] of the participants agreed to return to virtual theoretical activities and $6.09 \%$ [95 \% Cl: $2.89-12,34 \%$ ] to hospital practical activities. Only $66.09 \%$ had access to the Internet, a mobile device and an adequate study environment at home. Those who did not agree to return to the hospital practical activities had a history of a pathological condition $(p<0.001)$ and lived with two or more people over 65 years of age $(p<0.001)$. The main medical conditions of the relatives over 65 years of age were hypertension (36.89\%), diabetes $(19.04 \%)$ and obesity $(15.47 \%)$.

Conclusions: The number of midwifery students who agreed to return to the hospitals was low mainly because they had a history of a pathological condition and lived with relatives over 65 years of age. About half of the participants had digital resources that allowed them to access to remote learning.

Keywords: Coronavirus infections; Education, professional; Pandemics; Professional practice (Source: MeSH NLM).

1 Universidad Nacional Mayor de San Marcos, Escuela Profesional de Obstetricia, Centro Federado de Obstetricia. Lima, Perú.

2 Asociación Nacional de Estudiantes de Obstetricia del Perú. Lima, Perú.

3 Universidad Peruana Cayetano Heredia, Facultad de Salud Pública y Administración, Unidad de Informática Biomédica. Lima, Perú.

a Estudiante de Obstetricia.

b Egresado de la Maestría en Informática Biomédica.

*Autor corresponsal. 


\section{INTRODUCCIÓN}

La enfermedad por coronavirus (COVID-19) es originada por el virus SARS-CoV-2, el cual se caracteriza por una marcada respuesta inflamatoria. Los primeros casos fueron notificados a finales del 2019 , pero por su expansión ha alcanzado la categoría de pandemia ${ }^{(1-3)}$. Una respuesta inmediata de los gobiernos para evitar su rápido contagio fue establecer el distanciamiento social, el mismo que involucró el cese de múltiples actividades, entre las cuales se encuentra la educación superior ${ }^{(4)}$.

Sin embargo, la pandemia también ha favorecido el incremento de otras alternativas académicas, tales como la educación a distancia (teleconferencias), el uso de redes sociales y la práctica clínica mediante la telemedicina (5-7), las cuales han supuesto una redistribución de los recursos digitales en las universidades y la reevaluación de los métodos de enseñanza, aunque su implementación se está generando al mismo tiempo que su aplicación, con escasa evidencia al respecto ${ }^{(8)}$. Por otro lado, la práctica asistencial es la más afectada en relación con el aprendizaje clínico basado en competencias, puesto que se están suspendiendo o postergando múltiples rotaciones hasta el próximo año ${ }^{(9-11)}$.

Las investigaciones en la población estudiantil muestran su salud mental y el conocimiento que tienen de esta nueva enfermedad ${ }^{(12-14)}$. Sin embargo, los reportes acerca de la percepción del alumnado sobre el regreso a las actividades académicas son escasos, a pesar de que pueden ser útiles para plantear estrategias de solución por parte de las autoridades universitarias. Por ello, el objetivo de este estudio es determinar cuál es la perspectiva del estudiante de obstetricia acerca del retorno a las actividades teóricas y prácticas en la universidad en un contexto de pandemia.

\section{MATERIALES Y MÉTODOS}

\section{Diseño y población de estudio}

Se realizó un estudio de enfoque cuantitativo, observacional de tipo analítico transversal. La población estuvo conformada por estudiantes de obstetricia que fueron enrolados en la investigación durante el mes de abril del 2020. Los criterios de selección fueron los siguientes: estar matriculado al momento del estudio, ser mayor de edad y estar cursando el segundo, tercero, cuarto y quinto año académico (puesto que estos alumnos ya habrían asistido a la universidad al menos por un año).

El muestreo fue de tipo censal. Los participantes eran estudiantes de la Escuela Profesional de Obstetricia, considerada como la primera institución formativa en esta rama de Latinoamérica, y que pertenece a la Universidad Nacional Mayor de San Marcos (UNMSM) de Lima, Perú ${ }^{(15)}$. Los 429 estudiantes matriculados en la Escuela de Obstetricia fueron invitados a participar en la investigación. De ellos, 209 no respondieron el cuestionario y 105 no brindaron su consentimiento informado, aunque habían respondido el cuestionario. Por ello, solo el $26,80 \%(n=115)$ de los estudiantes fue parte del estudio.

\section{Variables y mediciones}

La variable principal, "Actividades universitarias", fue dimensionada en el retorno a las actividades teóricas virtuales y a las prácticas hospitalarias, situaciones en las que la posible predisposición del alumno pudiese ser "Estoy de acuerdo" o "No estoy de acuerdo" con el retorno a dichas actividades. Otra variable fueron los "recursos" con los que cuentan los estudiantes para poder reintegrarse a un sistema de educación remota, para lo que se consideraron las siguientes dimensiones: contar con acceso a internet, tener un dispositivo móvil y disponer de un ambiente para el estudio en el hogar. Como variables intervinientes se seleccionaron el lugar de residencia, su estado de salud, los antecedentes patológicos personales y el número de personas con las que convive (de ellas, se consultó cuántas son mayores de 65 años y cuáles eran sus antecedentes patológicos).

La técnica para recolección de información utilizada fue la encuesta, la cual fue difundida en un formato digital a fin de cumplir las restricciones de distanciamiento social que eviten el contagio del COVID-19. El instrumento de investigación fue desarrollado por los autores y validado mediante una evaluación de expertos (docentes de la Sección de Investigación de la Escuela Profesional de Obstetricia y obstetras investigadores del Colegio de Obstetras del Perú), con quienes se discutió el contenido del instrumento en reuniones virtuales. La consistencia interna fue evaluada en un estudio piloto con veinte estudiantes en el que utilizó la prueba alfa de Cronbach, con un resultado de 0,82 . Finalmente, el instrumento fue adaptado a un formato online mediante la plataforma Google Forms.

Los estudiantes de obstetricia fueron invitados a participar en la investigación por vía telefónica y se les especificó que se requería conocer su perspectiva respecto al retorno de las actividades universitarias. Se informó a los estudiantes de que solo los cuestionarios que contaban con el consentimiento informado se incluirían en el estudio.

\section{Análisis estadístico}

La herramienta de recolección de datos permitió exportar los registros en formato Excel. Primero, se evaluó la calidad de la información (ausencia de inconsistencias) y luego los registros fueron exportados al software STATA versión 14. Los datos descriptivos se reportaron con frecuencias y porcentajes, lo que generó, además, intervalos de confianza al $95 \%$ en las variables principales. Empleamos el programa Microsoft Word para elaborar las figuras.

Las asociaciones fueron evaluadas mediante un 
análisis crudo bivariado, con la prueba $X^{2}$ de Pearson. A continuación, el análisis ajustado fue evaluado mediante la prueba de regresión de Poisson. Se consideró como asociación estadísticamente significativa a todo valor de p menor a 0,05 .

\section{Consideraciones éticas}

Al inicio del cuestionario virtual se colocó un formato de consentimiento informado. Los registros que no tenían este requisito se excluyeron del estudio. Asimismo, la información sobre la identidad de los participantes fue obviada.

\section{RESULTADOS}

En el estudio participaron 115 estudiantes de obstetricia de una universidad pública peruana, de los cuales el 97,39\% reside en Lima. El 58,2 6\% [IC95\%: 48,93-67,03 \%] refiere estar de acuerdo con el inicio de las clases teóricas virtuales, mientras que el 6,09\% [IC95\%: 2,89-12,34 \%] concuerda con el retorno de las prácticas hospitalarias. Asimismo, el $94,78 \%$ reportó estar sano. Los antecedentes patológicos más prevalentes fueron el asma $(6,96 \%)$ y alguna enfermedad mental (3,48\%). Finalmente, el 48,7\% vive con al menos una persona mayor de 65 años en su hogar (Tabla 1).

Tabla 1. Características de los estudiantes de obstetricia de una universidad pública de Lima, 2020

\begin{tabular}{|c|c|c|}
\hline Características & N & $(\%)$ \\
\hline \multicolumn{3}{|l|}{ ¿Reside en Lima? } \\
\hline Sí & 112 & 97,39 \\
\hline No & 3 & 2,61 \\
\hline \multicolumn{3}{|c|}{ ¿Está de acuerdo con el retorno de las clases teóricas virtuales? } \\
\hline Sí & 67 & 58,26 \\
\hline No & 48 & 41,74 \\
\hline \multicolumn{3}{|c|}{ ¿Está de acuerdo con el retorno de las prácticas hospitalarias? } \\
\hline Sí & 7 & 6,09 \\
\hline No & 108 & 93,91 \\
\hline \multicolumn{3}{|c|}{ Actualmente, ¿se encuentra bien de salud? } \\
\hline Sí & 109 & 94,78 \\
\hline \multicolumn{3}{|l|}{ No } \\
\hline Ansiedad y depresión & 2 & 1,74 \\
\hline Asma & 1 & 0,87 \\
\hline Migraña & 1 & 0,87 \\
\hline Resfriado & 2 & 1,74 \\
\hline \multicolumn{3}{|l|}{ Antecedente de asma } \\
\hline Sí & 8 & 6,96 \\
\hline No & 107 & 93,04 \\
\hline \multicolumn{3}{|c|}{ Antecedente de enfermedad mental } \\
\hline Sí & 4 & 3,48 \\
\hline No & 111 & 96,52 \\
\hline \multicolumn{3}{|c|}{ Antecedente de hipertensión arterial } \\
\hline Sí & 1 & 0,87 \\
\hline No & 114 & 99,13 \\
\hline \multicolumn{3}{|c|}{ Antecedente de obesidad } \\
\hline Sí & 3 & 2,61 \\
\hline No & 112 & 97,39 \\
\hline
\end{tabular}




\section{Características}

Número de personas con las que convive en el hogar

Menos de dos personas

Tres a cinco personas

Más de cinco personas

Número de personas mayores de 65 años con las que convive

Una persona

Dos personas

Más de dos personas

Ninguna

Total
N

(\%)

5,22

60,87

33,91

39

34,78

13,04

0,87

51,30

100
En la figura 1 se muestra la cantidad de estudiantes que cuentan con los recursos necesarios para el retorno de las actividades académicas en un contexto de COVID-19. De todos los participantes, 107 tenían un dispositivo electrónico (93,04\%): de este grupo, 98 estudiantes
$(91,59 \%)$ contaban con acceso a internet; y de ellos, $76(77,55 \%)$ disponía de un ambiente adecuado para el estudio en su hogar. Finalmente, el $66,09 \%$ contaba con los tres recursos referidos.

\section{Estudiantes de obstetricia enrolados}

$\mathrm{n}=115$

Tiene un dispositivo que le permite acceder a internet
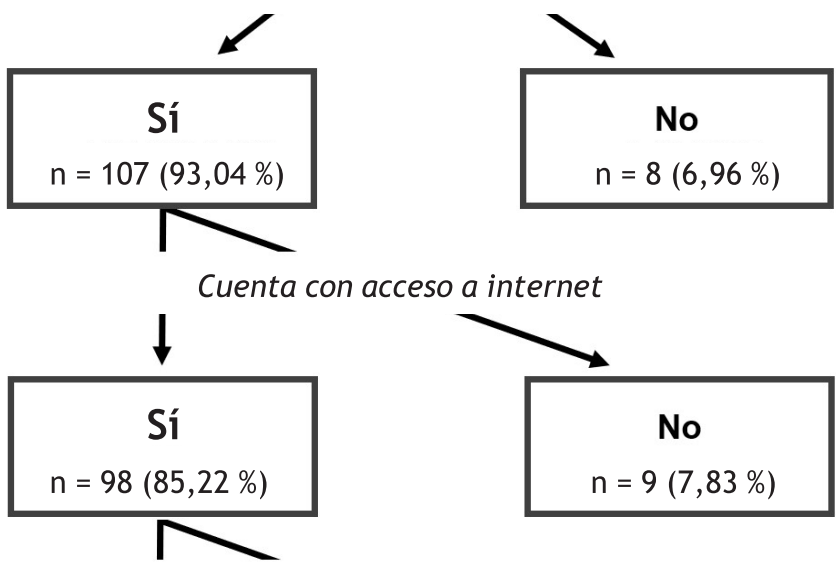

Dispone de un ambiente adecuado para el estudio
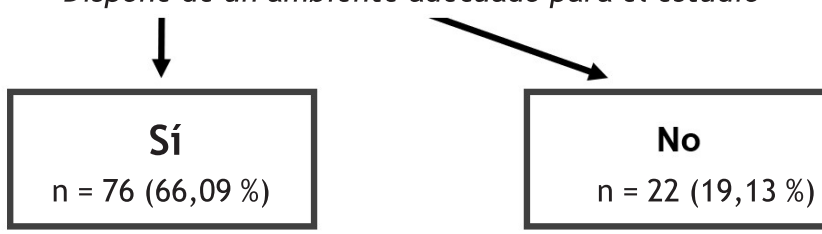

Figura 1. Recursos disponibles en los estudiantes de obstetricia de una universidad pública, 2020 
En el grupo que estaba de acuerdo con el retorno de las clases teóricas virtuales, un $94,03 \%$ contaba con acceso a internet y un 17,91\% convivía con más de 2 personas mayores de 65 años en el hogar, aunque ninguna característica alcanzó la significancia estadística. Por otro lado, la autopercepción del estudiante de encontrarse bien de salud $(p<0,001)$, vivir con más de tres personas $(p<0,001)$ y contar con un dispositivo móvil que permita acceder a internet $(p<0,001)$ fueron características de quienes estuvieron de acuerdo con el retorno de las prácticas hospitalarias. Mientras que el estar en desacuerdo es más evidente en quienes residen en Lima $(p=0,039)$, presentan un antecedente patológico $(p<0,001)$ y conviven con dos o más personas mayores de 65 años $(p<0,001)$ (Tablas 2 y 3$)$.

Tabla 2. Características asociadas al retorno de las clases teóricas virtuales en tiempos de COVID-19

\begin{tabular}{|c|c|c|c|c|c|c|}
\hline \multirow[b]{2}{*}{ Características } & \multicolumn{4}{|c|}{ Retorno de las clases teóricas virtuales } & \multirow[t]{2}{*}{$p^{\dagger}$} & \multirow[t]{2}{*}{$p^{\pi}$} \\
\hline & $\mathbf{N}$ & $(\%)$ & N & (\%) & & \\
\hline \multicolumn{7}{|l|}{ ¿Reside en Lima? } \\
\hline Sí & 66 & 98,51 & 46 & 95,83 & 0,489 & 0,462 \\
\hline No & 1 & 1,49 & 2 & 4,17 & & \\
\hline \multicolumn{7}{|c|}{ Actualmente, ¿se encuentra bien de salud? } \\
\hline Sí & 65 & 97,07 & 44 & 91,67 & 0,320 & 0,358 \\
\hline No & 2 & 2,99 & 4 & 8,33 & & \\
\hline \multicolumn{7}{|l|}{ Antecedente patológico } \\
\hline Sí & 9 & 13,49 & 10 & 20,83 & 0,343 & 0,636 \\
\hline No & 58 & 86,57 & 38 & 79,17 & & \\
\hline \multicolumn{7}{|c|}{ Número de personas con las que convive en el hogar } \\
\hline Menos de dos personas & 3 & 4,48 & 3 & 6,25 & Ref. & Ref. \\
\hline Tres a cinco personas & 42 & 62,69 & 28 & 58,33 & 0,665 & 0,602 \\
\hline Más de cinco personas & 22 & 32,84 & 17 & 35,42 & 0,781 & 0,889 \\
\hline \multicolumn{7}{|c|}{ Número de personas mayores de 65 años con las que convive } \\
\hline Ninguna & 33 & 49,25 & 26 & 54,17 & Ref. & Ref. \\
\hline Una persona & 22 & 32,84 & 18 & 37,50 & 0,927 & 0,936 \\
\hline Dos o más personas & 12 & 17,91 & 4 & 8,33 & 0,114 & 0,174 \\
\hline \multicolumn{7}{|c|}{ ¿Cuenta con dispositivo móvil que permite acceder a internet? } \\
\hline Sí & 62 & 92,54 & 45 & 93,75 & 0.792 & 0.375 \\
\hline No & 5 & 7,46 & 3 & 6,25 & & \\
\hline \multicolumn{7}{|c|}{ ¿Cuenta con acceso a internet? } \\
\hline Sí & 63 & 94,03 & 40 & 83,33 & 0.146 & 0.121 \\
\hline No & 4 & 5,97 & 8 & 16,67 & & \\
\hline \multicolumn{7}{|c|}{ ¿Cuenta con un ambiente de estudio? } \\
\hline Sí & 55 & 82,09 & 35 & 72,92 & 0,284 & 0,342 \\
\hline No & 12 & 17,91 & 13 & 27,08 & & \\
\hline Total & 67 & 100 & 48 & 100 & & \\
\hline
\end{tabular}

† Análisis crudo: Evaluado mediante la prueba $X^{2}$ de Pearson

†† Análisis ajustado: Evaluado mediante la prueba de regresión de Poisson 
Tabla 3. Características asociadas al retorno de las prácticas hospitalarias en tiempos de COVID-19

\begin{tabular}{|c|c|c|c|c|c|c|}
\hline \multirow[b]{2}{*}{ Caracteristicas } & \multicolumn{4}{|c|}{ Retorno de las prácticas hospitalarias } & \multirow[t]{2}{*}{$p^{\dagger}$} & \multirow[t]{2}{*}{$p^{\pi}$} \\
\hline & $\mathbf{N}$ & $(\%)$ & $\mathbf{N}$ & $(\%)$ & & \\
\hline \multicolumn{7}{|l|}{ ¿Reside en Lima? } \\
\hline Sí & 6 & 85,71 & 106 & 98,15 & 0,045 & 0,039 \\
\hline No & 1 & 14,29 & 2 & 1,85 & & \\
\hline \multicolumn{7}{|c|}{ Actualmente, ¿se encuentra bien de salud? } \\
\hline Sí & 7 & 100 & 102 & 94,44 & 0,522 & $<0,001$ \\
\hline No & 0 & 0,00 & 6 & 5,56 & & \\
\hline \multicolumn{7}{|l|}{ Antecedente patológico } \\
\hline Sí & 0 & 0,00 & 19 & 17,59 & 0,225 & $<0.001$ \\
\hline No & 7 & 100 & 89 & 82,41 & & \\
\hline \multicolumn{7}{|c|}{ Número de personas con las que convive en el hogar } \\
\hline Menos de dos personas & 0 & 0,00 & 6 & 5,56 & Ref. & Ref. \\
\hline Tres a cinco personas & 4 & 57,14 & 66 & 61,11 & 0,579 & $<0,001$ \\
\hline Más de cinco personas & 3 & 42,86 & 36 & 33,33 & 0,469 & $<0,001$ \\
\hline \multicolumn{7}{|c|}{ Número de personas mayores de 65 años con las que convive } \\
\hline Ninguna & 4 & 57,14 & 55 & 50,93 & Ref. & Ref. \\
\hline Una persona & 3 & 42,86 & 37 & 34,26 & 0,891 & 0,948 \\
\hline Dos o más personas & 0 & 0,00 & 16 & 14,81 & 0,469 & $<0,001$ \\
\hline \multicolumn{7}{|c|}{ ¿Cuenta con dispositivo móvil que permite acceder a internet? } \\
\hline Sí & 7 & 100 & 100 & 92,59 & 0,455 & $<0,001$ \\
\hline No & 0 & 0,00 & 8 & 7,41 & & \\
\hline \multicolumn{7}{|c|}{ ¿Cuenta con acceso a internet? } \\
\hline Sí & 6 & 85,71 & 97 & 89,81 & 0,731 & 0,770 \\
\hline No & 1 & 14,29 & 11 & 10,19 & & \\
\hline \multicolumn{7}{|c|}{ ¿Cuenta con un ambiente de estudio? } \\
\hline Sí & 6 & 85,71 & 84 & 77,78 & 0,630 & 0,674 \\
\hline No & 1 & 14,29 & 24 & 22,22 & & \\
\hline Total & 7 & 100 & 108 & 100 & & \\
\hline
\end{tabular}

† Análisis crudo: Evaluado mediante la prueba $X^{2}$ de Pearson

†† Análisis ajustado: Evaluado mediante la prueba regresión de Poisson

En la tabla 4 se muestran los antecedentes clínicos de los obesidad $(26,67 \%)$ y diabetes $(20,04 \%)$. Mientras que familiares que conviven los estudiantes de obstetricia. los mayores de 65 años presentaron hipertensión arterial En el grupo de menores de 65 años, los antecedentes (36,89\%), diabetes $(19,04 \%)$ y obesidad $(15,47 \%)$. más recurrentes fueron hipertensión arterial (31,67\%), 
Tabla 4. Antecedentes clínicos de los familiares con quienes conviven los estudiantes de obstetricia de una universidad pública, 2020

\begin{tabular}{|c|c|c|}
\hline Características & $\mathrm{N}$ & $(\%)$ \\
\hline \multicolumn{3}{|c|}{ Antecedentes clínicos de familiares con menos de 65 años $(n=60)$} \\
\hline Alteraciones con la tiroides & 1 & 1,67 \\
\hline Anemia & 1 & 1,67 \\
\hline Artritis & 1 & 1,67 \\
\hline Asma & 1 & 1,67 \\
\hline Bronquiectasia & 1 & 1,67 \\
\hline Cáncer & 1 & 1,67 \\
\hline Diabetes & 12 & 20,04 \\
\hline Enfermedad mental & 4 & 6,67 \\
\hline Gastritis & 1 & 1,67 \\
\hline Hipertensión arterial & 19 & 31,67 \\
\hline Hipertiroidismo & 1 & 1,67 \\
\hline Obesidad & 16 & 26,67 \\
\hline Osteoporosis & 1 & 1,67 \\
\hline \multicolumn{3}{|c|}{ Antecedentes clínicos de familiares con 65 años o más $(n=84)$} \\
\hline Artritis & 1 & 1,19 \\
\hline Asma & 4 & 4,76 \\
\hline Bronquiectasia & 1 & 1,19 \\
\hline Cáncer & 1 & 1,19 \\
\hline Cataratas & 1 & 1,19 \\
\hline Cardiopatía & 1 & 1,19 \\
\hline Depresión & 3 & 3,57 \\
\hline Diabetes & 16 & 19,04 \\
\hline Fibrosis pulmonar & 1 & 1,19 \\
\hline Gastritis & 2 & 2,38 \\
\hline Hipertensión arterial & 31 & 36,89 \\
\hline Obesidad & 13 & 15,47 \\
\hline Osteoporosis & 3 & 3,57 \\
\hline Síndrome de Parkinson & 2 & 2,38 \\
\hline Trombocitopenia & 1 & 1,19 \\
\hline Hipotiroidismo & 3 & 3,57 \\
\hline
\end{tabular}

Puede haber personas que presenten más de un antecedente a la vez Antecedentes autoreportados

\section{DISCUSIÓN}

Determinamos que cerca de la mitad de los participantes están dispuestos a retornar sus estudios mediante clases teóricas virtuales, posiblemente por las limitaciones que presenta la educación remota, como la necesidad de dispositivos tecnológicos o conectividad a internet en adecuadas condiciones, la escasa retroalimentación durante clases asincrónicas, la inexperiencia de algunos docentes para adaptarse a entornos virtuales, entre otras ${ }^{(16,17)}$. Además, se halló que muy pocos estudiantes están dispuestos a retomar la práctica hospitalaria debido al riesgo que implica. Este resultado es similar al que reportaron los internos de medicina peruanos, de los cuales, el $78 \%$ estuvo de acuerdo en retirarse de sus sedes hospitalarias debido a la pandemia (18).

Por otro lado, casi la mitad de los estudiantes contaban con los elementos necesarios para incorporarse a una educación remota (ambiente, equipo e internet): esto refleja la desventaja que podría tener un grupo de alumnos para adaptarse al cambio tecnológico ${ }^{(19)}$. Este problema ha 
sido afrontado de diversas formas, por ejemplo, el Reino Unido abrió las bibliotecas bajo ciertas restricciones de seguridad, para generar espacios óptimos de aprendizaje ${ }^{(20)}$, mientras que en Singapur se gestionó la distribución de computadoras portátiles para los estudiantes ${ }^{(21)}$.

Un numeroso grupo de los participantes que no estuvieron de acuerdo con retornar a sus prácticas hospitalarias vivían con dos o más personas mayores de 65 años y contaban con un antecedente patológico por lo menos. Esto evidencia una constante preocupación del alumno, pues asume que podría enfermar y también contagiar a su familia (22,23). En México se ha observado una situación similar, incluso se propuso que quienes no presenten factores de riesgo puedan ser reubicados en instituciones médicas. Esto no ha sido aceptado por los estudiantes, pues ellos perciben que no existen garantías de seguridad personal, así como tampoco tienen la certeza de ser ubicados en servicios con bajo riesgo de contagio ${ }^{(24,25)}$.

Al evaluar las características de las familias de los estudiantes, encontramos que, aproximadamente, la mitad de ellos convive con al menos un familiar mayor a 65 años, lo cual, según diversos autores, es un factor de riesgo para desarrollar un cuadro grave o de morir por COVID-19 (26-29). Hallamos que esta población vulnerable tenía comorbilidades como hipertensión, diabetes y obesidad, que favorecen un cuadro severo de COVID-19 ${ }^{(30-33)}$. Esto sugiere que se debe considerar la protección adecuada del estudiante para evitar múltiples focos de infección en cada uno de sus hogares.

Es preciso que los resultados se circunscriban a ciertas limitaciones del estudio. Debido a la cuarentena, los datos se recolectaron de forma virtual, lo que puede subestimar a los estudiantes que carecen de conexión remota, y que por esta razón no hayan participado. Por otro lado, esta investigación es la primera que reporta la percepción del estudiante de obstetricia en el entorno latinoamericano, y que incluye, además, un análisis de los recursos con los que cuenta este grupo y de su entorno familiar como factores que influyen en la decisión de optar por el retorno a las actividades universitarias.

Concluimos que la proporción de estudiantes a favor del retorno a los hospitales es baja, debido a tienen antecedentes patológicos personales y a que conviven con familiares mayores de 65 años. Además, cerca de la mitad de los participantes cuentan con recursos que les facilita el aprendizaje remoto.

Contribuciones de autoría: JRV, DCG, VDO Y JRS participaron en la conceptualización, metodología, investigación, administración del proyecto, redacción, revisión y aprobación de la versión final. VMA participó en la asesoría metodológica, análisis formal, curaduría de datos, supervisión, redacción, revisión y aprobación de la versión final.

Fuentes de financiamiento: El artículo ha sido financiado por los autores.

Conflicto de interés: Los autores declaran no tener ningún conflicto de interés.

\section{REFERENCIAS BIBLIOGRÁFICAS}

1. Accinelli RA, Zhang Xu CM, Ju Wang J-D, Yachachin-Chávez JM, Cáceres-Pizarro JA, Tafur-Bances KB, et al. COVID-19: la pandemia por el nuevo virus SARS-CoV-2. Rev Peru Med Exp Salud Pública. 2020; 37(2): 302-11.

2. Patel RH. Clinical outcomes and prognosis of patients with HIV and SARS-CoV-2 coinfection. J Med Virol. 2021; 93(1): 105-6.

3. Sarvepalli D. Coronavirus Disease 2019: A Comprehensive Review of Etiology, Pathogenesis, Diagnosis, and Ongoing Clinical Trials. Cureus. 2020; 12(5): e8076.

4. Tabari P, Amini M, Moghadami M, Moosavi M. International Public Health Responses to COVID-19 Outbreak: A Rapid Review. Iran J Med Sci. 2020; 45(3): 157-69.

5. Sklar DP. COVID-19: Lessons From the Disaster That Can Improve Health Professions Education. Acad Med J Assoc Am Med Coll. 2020; 95(11): 1631-3.

6. Kinder F, Harvey A. Covid-19: the medical students responding to the pandemic. BMJ Student. 2020; 369: m2160.

7. Dedeilia A, Sotiropoulos MG, Hanrahan JG, Janga D, Dedeilias P, Sideris M. Medical and Surgical Education Challenges and Innovations in the COVID-19 Era: A Systematic Review. In Vivo. 2020; 34(3 Suppl): 1603-11.

8. Raymond-Hayling 0 . What lies in the year ahead for medical education? A medical student's perspective during the COVID-19 pandemic. Med Educ Online. 2020; 25(1):1781749.

9. Roskvist R, Eggleton K, Goodyear-Smith F. Provision of e-learning programmes to replace undergraduate medical students' clinical general practice attachments during COVID-19 stand-down. Educ Prim Care 2020; 31(4): 247-54.

10. Guadix SW, Winston GM, Chae JK, Haghdel A, Chen J, Younus I, et al. Medical Student Concerns Relating to Neurosurgery Education During COVID-19. World Neurosurg. 2020; 139: e836-47.

11. Chang WJ, Jiang YD, Xu JM. Experience of teaching and training for medical students at gastrointestinal surgery department under COVID-19 epidemic situation. Zhonghua Wei Chang Wai Ke Za Zhi. 2020; 23(6): 616-8.

12. Sögüt S, Dolu I, Cangöl E. The relationship between COVID-19 knowledge levels and anxiety states of midwifery students during the outbreak: A cross-sectional web-based survey. Perspect Psychiatr Care. 2021; 57(1): 246-52.

13. Mechili EA, Saliaj A, Kamberi F, Girvalaki C, Peto E, Patelarou AE, et al. Is the mental health of young students and their family members affected during the quarantine period? Evidence from the COVID-19 pandemic in Albania. J Psychiatr Ment Health Nurs. 2020.

14. Awingura-Apanga P, Kamar-Lettor IB, Akunvane R. Practice of COVID-19 Preventive Measures and Its Associated Factors among Students in Ghana. Am J Trop Med Hyg. 2020; 104(2): 526-31.

15. Universidad Nacional Mayor de San Marcos. Descripción de la Escuela Profesional de Obstetricia [Internet]. Escuela Profesional de Obstetricia. 2020. Disponible en: https://medicina.unmsm.edu.pe/ index.php/en/descripcion-de-la-escuelavv 
16. Gallo N. Acerca de los estudiantes que transitan nuestras aulas en tiempos de pandemia [Internet]. Universidad Nacional de Río Cuarto. 2020. Disponible en:https://www.evelia.unrc.edu.ar/evelia/portal/files/articulosAulasExtendidas Acercadelosestudiantesquetransitannuestrasaulasentiemposdepandemia.pdf

17. Herrera-Añazco P, Toro-Huamanchumo CJ. Educación médica durante la pandemia de COVID-19: iniciativas mundiales para el pregrado, internado y residentado médico. Acta Méd Perú. 2020; 37(2): 169-75.

18. Albitres-Flores L, Pisfil-Farroñay YA,, Guillen-Macedo K, Niño-García R, Alarcón-Ruiz CA. Percepción de los internos de medicina sobre el internado durante la pandemia por COVID-19 en Perú. Rev Perú Med Exp Salud Pública. 2020; 37(3): 504-9.

19. McMaster D, Veremu M, Santucci C. COVID-19: Opportunities for professional development and disruptive innovation. The C y T. 2020; 17(1): 238-240.

20. Luyben A, Fleming V, Vermeulen J. Midwifery education in COVID-19time: Challenges and opportunities. Midwifery. 2020; 89: 102776.

21. Lim M. Educating despite the Covid-19 outbreak: lessons from Singapore [Internet]. Reino unido: Times Higher Education. 2020. Disponible en: https: / / www.timeshighereducation.com/blog/educating-despitecovid-19-outbreak-lessons-singapore\#

22. Wang C, Pan R, Wan X, Tan Y, Xu L, Ho CS, et al. Immediate psychological responses and associated factors during the initial stage of the 2019 Coronavirus Disease (COVID-19) epidemic among the general population in China. Int J Environ Res Public Health. 2020; 17(5): 1729.

23. Li Y, Wang $Y$, Jiang J, Valdimarsdóttir UA, Fall $\mathrm{K}$, Song $\mathrm{H}$, et al. Psychological distress among health professional students during the COVID-19 outbreak. Psychol Med. 2020: 1-3.

24. Gobierno de México. Médicos e internos de pregrado y pasantes de enfermería, sin factores de riesgo, podrán reincorporándose voluntariamente a unidades médicas [Internet]. Ciudad de México: Instituto de Seguridad y Servicios Sociales de los Trabajadores del Estado. 2020. Disponible en: https://www.gob.mx/issste/prensa/ medicos-internos-de-pregrado-y-pasantes-de-enfermeria-sin-factoresde-riesgo-podran-reincorporarse-voluntariamente-a-unidades-medicasissste?idiom=es

25. Arista L. Entre miedo y amenazas médicos internos regresan a hospitales [Internet]. Ciudad de México: Expansión política. 2020. Disponible en: https://politica.expansion.mx/mexico/2020/05/14/entre-miedo-yamenazas-medicos-internos-regresan-a-hospitales

26. Zhou F, Yu T, Du R, Fan G, Liu Y, Liu Z, et al. Clinical course and risk factors for mortality of adult inpatients with COVID-19 in Wuhan, China: a retrospective cohort study. Lancet. 2020; 395(10229): 1054-62.

27. Acosta G, Escobar G, Bernaola G, Alfaro J, Taype W, Marcos C, et al. Caracterización de pacientes con COVID-19 grave atendidos en un hospital de referencia nacional del Perú. Rev Peru Med Exp Salud Pública. 2020; 37(2): 253-8.

28. Escobar G, Matta J, Ayala R, Amado J. Características clinicoepidemiológicas de pacientes falecidos por COVID-19 em um hospital nacional de Lima, Perú. Rev Fac Med Hum. 2020; 20(2): 180-5.

29. Mejía F, Medina C, Cornejo E, Morello E, Vásquez S, Alave J, et al. Características clínicas y factores associados a mortalidade em pacientes adultos hospitalizados por COVID-19 em um hospital público de Lima, Perú. Scielo Preprint. 2020.
30. Fang L, Karakiulakis G, Roth $M$. Are patients with hypertension and diabetes mellitus at increased risk for COVID-19 infection?. Lancet Respir Med. 2020; 8(4): e21.

31. Li B, Yang J, Zhao F, Zhi L, Wang X, Liu L, et al. Prevalence and impact of cardiovascular metabolic diseases on COVID-19 in China. Clin Res Cardiol. 2020; 109(5): 531-8.

32. Schiffrin EL, Flack JM, Ito S, Muntner P, Webb RC. Hypertension and COVID-19. Am J Hypertens. 2020; 33(5): 373-4.

33. Li X, Xu S, Yu M, Wang K, Tao Y, Zhou Y. Risk factors for severity and mortality in adult COVID-19 inpatients in Wuhan. J Allergy Clin Immunol. 2020; 146(1): 110-8.

\section{Correspondencia:}

Víctor Hugo Moquillaza Alcántara

Dirección: Condominio Los Nogales, edificio 8, departamento 503. Urbanización Los Parques de El Agustino. El Agustino, Lima, Perú.

Teléfono: 051982065404

Correo electrónico: victor.moquillaza@upch.pe

Recibido: 16 de diciembre de 2020

Evaluado: 28 de enero de 2021

Aprobado: 26 de febrero de 2021

(c) La revista. Publicado por Universidad de San Martín de Porres, Perú. (c) Br Licencia de Creative Commons Artículo en acceso abierto bajo términos de Licencia Creative Commons Atribución 4.0 Internacional. (http://creativecommons.org/licenses/by/4.0/)

\section{ORCID iDs}

Jennifer Rojas-Vega

Diayan Castro-Gómez

(ㄱ) https: / / orcid.org/0000-0002-3205-1633

Valery Damacén-Oblitas

(1) https: / / orcid.org/0000-0003-4054-3061

Jessica Rojas-Silva

(1) https: / / orcid.org/0000-0002-8835-7543

Victor Moquillaza-Alcántara
(1) https: / / orcid.org/0000-0002-0718-7272 